\title{
Violência obstétrica: fatores desencadeantes e medidas preventivas de enfermagem
}

RESUMO | OBJETIVO: Caracterizar os fatores que ocasionam a violência obstétrica e a importância da enfermagem no desenvolvimento de medidas preventivas. MÉTODOS: Trata-se de uma revisão sistemática da literatura com metassíntese, com utilização do protocolo PRISMA. Realizou-se a pesquisa referida na fonte de busca: Biblioteca Virtual em Saúde (BVS) e nas bases de dados: SCIELO e LILACS, apresentando uma totalidade de 61 documentos. Após estabelecimento e aplicação dos critérios de inclusão: artigos disponíveis gratuitos, publicados entre 2015 a 2020, em língua portuguesa, inglesa e espanhola e exclusão: não apresentassem à temática, repetitivos, dissertações e resumos, resultando em um total de 10 artigos. RESULTADOS: O processo do parto é um acontecimento repleto de possíveis equívocos, condutas dolorosas e negligências, que podem gerar a violência obstétrica causando traumas físicos e psicológicos irreversíveis. CONCLUSÃO: Por meio desse estudo buscou destacar a importância de minimizar as praticas abusiva, com o uso de estratégias que efetivem programas e políticas voltadas ao binômio mãe-filho.

Palavras-chaves: Violência Obstétrica. Assistência de Enfermagem. Saúde da Mulher.

\begin{abstract}
OBJECTIVE: To characterize the factors that cause obstetric violence and the importance of nursing in the development of preventive measures. METHODS: This is a systematic review of the literature with metasynthesis, using the PRISMA protocol. The research was reported in the search source: Virtual Health Library (VHL) and in the databases: SCIELO and LILACS, presenting a total of 61 documents. After establishment and application of inclusion criteria: free available articles, published between 2015 and 2020, in Portuguese, English and Spanish and exclusion: do not present to the theme, repetitive, dissertations and abstracts, resulting in a total of 10 articles. RESULTS: The delivery process is an event full of possible misunderstandings, painful behaviors and negligence, which can generate obstetric violence causing irreversible physical and psychological trauma. CONCLUSION: Through this study, we sought to highlight the importance of minimizing abusive practices, with the use of strategies that effectively implement programs and policies aimed at the mother-child binomial.
\end{abstract}

Keywords: Obstetric violence. Nursing care. Women's Health.

RESUMEN | OBJETIVO: Caracterizar los factores que causan la violencia obstétrica y la importancia de la enfermería en el desarrollo de medidas preventivas. METODOS: Se trata de una revisión sistemática de la literatura con metasíntesis, utilizando el protocolo PRISMA. La investigación fue reportada en la fuente de búsqueda: Virtual Health Library (VHL) y en las bases de datos: SCIELO y LILACS, presentando un total de 61 documentos. Tras el establecimiento y aplicación de criterios de inclusión: artículos gratuitos, publicados entre 2015 y 2020, en portugués, inglés y español y exclusión: no se presentan al tema, repetitivos, disertaciones y resúmenes, dando como resultado un total de 10 artículos. RESULTADOS: El proceso de entrega es un evento lleno de posibles malentendidos, comportamientos dolorosos y negligencia, que puede generar violencia obstétrica causando traumas físicos y psicológicos irreversibles. CONCLUSION: A través de este estudio, buscamos destacar la importancia de minimizar las prácticas abusivas, con el uso de estrategias que implementen eficazmente programas y políticas dirigidas al binomio materna-infantil.

Palabras claves: Violencia obstétrica. Cuidado de enfermería. Salud de la Mujer.

\section{Maria Patrícia Vitorino de Sousa}

Acadêmica de Enfermagem do Centro Universitário de Juazeiro do Norte- CE (UNIJUAZEIRO). Pós-graduanda em Enfermagem em pediatria e saúde mental (FAVENI).

ORCID: 0000-0001-7159-7551

\section{Lohany Stéfhany Alves dos Santos}

Acadêmica de Enfermagem do Centro Universitário de Juazeiro do Norte- CE (UNIJUAZEIRO). Pós- graduanda em Docência do ensino superior e Enfermagem em UTI (FAVENI).

ORCID: 0000-0003-3440-740X

\section{Geovanna Renaissa Ferreira Caldas}

Acadêmica de Enfermagem do Centro Universitário de Juazeiro do Norte- CE (UNIJUAZEIRO).

ORCID: 0000-0001-9820-309X
Francisco de Assis Moura Batista

Acadêmico de Enfermagem do Centro Universitário de Juazeiro do Norte- CE (UNIJUAZEIRO). Pós-graduando em Saúde Pública com Ênfase em Estratégia de Saúde da Família (FAVENI).

ORCID: 0000-0003-2403-4830

\section{Cicero Rafael Lopes da Silva}

Enfermeiro. Especialista em Enfermagem Dermatológica, Pós graduando em Docência do Ensino Superior - UNIJUAZEIRO e em Gerontologia e saúde do idoso - FAVENI, Docente da graduação em Enfermagem - UNIJUAZEIRO.

ORCID: 0000-0001-8819-5380

Recebido em: 21/11/2020

Aprovado em: 03/02/2021
INTRODUÇÃO

$\Lambda$ experiência do parto foi considerado um momento importante na vida das mulheres, permitindo a transição para um novo papel social: ser mãe ${ }^{1}$. Até meados do século XIX, os partos aconteciam no domicilio,sendo assistida por parteiras, que lutavam para tornar esse momento confortável e alegre².

A institucionalização do parto no século XX levou ao uso de tecnologias durante o cuidado, em situações classificadas como de alto risco para mãe e fiIho, assim houve uma redução nas taxas de mortalidade materna e neonatal, no entanto, essas práticas tornaram-se meca- 
nizadas, fragmentadas e desumanizadas, devido ao excesso de intervenções desnecessárias, diminuindo a autonomia das mulheres no momento do parto ${ }^{3-4}$.

O termo violência obstétrica tem sido utilizado desde o reconhecimento dessa violação à mulher, sendo definido como uma apropriação do corpo feminino e sua autonomia reprodutiva, durante o processo pré-parto, parto e pós-parto, por profissionais de saúde, expondo as mulheres a comportamentos desumanizados, usam de procedimentos dolorosos ou embaraçosos, bem como drogas desnecessárias, substituindo o processo natural do nascimento em patológico e usando atitudes abusivas em relação ao estado psicológico das mulheres ${ }^{5}$.

Uma estudo realizado no Brasil, em 2015 com 603 puérperas revelou índices bastante altos com relação as intervenções $(86,5 \%)$ sofreu algum tipo de intervenção durante o parto ${ }^{7}$. Algumas intervenções comuns são a manobra de kristeller (37\%), a episiotomia (56\%) e uso de ocitocina de rotina e amniotomia $(40 \%) 6,7,8$.esse estudo tem um contexto de suma relevância, pois é composto pelas altas taxas de intervenções empregadas na atenção ao parto e ao nascimento que ficam bem evidenciados com os dados apresentados ${ }^{6}$.

É notável o desempenho da equipe de saúde na redução da violência no campo obstétrico, devido à sua ação direta durante as fases clínicas do processo de pré-parto, parto e puerpério, vaginal e cesariana, que proporcionam as mulheres e seus familiares, cuidados integrais e humanizados ${ }^{7}$.

Destaca-se ainda que a prática assistencial da equipe de enfermagem é um dos fatores que vem desenvolvendo uma assistência humanizada, o que justifica a realização deste estudo uma vez que cardoso8 ressalta que a enfermagem também é uma peça importante para acolher e humanizar as gestantes no processo parir/nascer. Assim, compreende-se que a atenção obstétrica e neonatal, realizada pelos serviços de saúde, deve ter como características essenciais à qualidade e a humanização ${ }^{9}$.

Dada a importância da equipe de enfermagem no combate à violência no campo obstétrico, bem como a importância do assunto discutido, foi feita a seguinte pergunta: Quais os fatores desencadeiam a violência obstétrica?

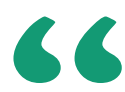

É notável o desempenho da equipe de saúde na redução da violência no campo obstétrico, devido à sua ação direta durante as fases clínicas do processo de pré-parto, parto e puerpério, vaginal e cesariana, que proporcionam as mulheres e seus familiares, cuidados integrais e humanizados
Em virtude disso, o objetivo do estudo foi caracterizar os fatores que ocasionam a violência obstétrica e a importância da enfermagem no desenvolvimento de medidas preventivas.

\section{METODO}

Trata-se de uma revisão sistemática da literatura com metassíntese. Esse tipo de pesquisa busca proporcionar um $\mathrm{CO}^{-}$ nhecimento lógico e cientifico, através de objetivos estabelecidos pelos autores e o uso da metodologia para a obtenção de resultados com altos níveis de evidências, por intermeio da metassíntese, utilizada para associar estudos qualitativos, localizando e dividindo-se em temas, áreas ou tópico. A abordagem qualitativa segue o raciocínio de investigar, avaliar e interpretar os dados ${ }^{10-11}$.

Realizou-se a pesquisa referida na fonte de busca: Biblioteca Virtual em Saúde (BVS) e nas bases de dados: ScientificElectronic Library Online (SciELO) e Literatura Latino-americana e do Caribe em Ciências da Saúde (LILACS), por intermeio da utilização dos descritores em ciências da saúde (DeCS): "Violência Obstétrica", "Assistência de Enfermagem", em associação do operador booleano AND.

Dentre os critérios de inclusão, citam-se os estudos: disponíveis integralmente de forma gratuita, publicados entre 2015 a 2020, publicados em português, inglês ou espanhol. Os critérios de exclusão definiram-se em estudos que não apresentassem a temática central, que não respondessem a questão norteadora, repetitivos, dissertações, teses, documentários, monografias, projetos, resumos simples ou expandidos. Após a pesquisa e aplicação dos filtros de ano e linguagem, realizou-se leitura dos resumos, para meIhor selecionar os que se enquadravam nos critérios.

A resolução sinaliza para as pesquisas que não precisam ser registradas e avaliadas pelo sistema CEP/CONEP. De acordo com Carvalho $(2017)^{12}$, a definição da na- 
tureza dos dados de pesquisa mostra-se como critério para definir a exigibilidade da avaliação dos projetos no âmbito das ciências humanas e sociais. São exem- plos as investigações cujos participantes não são identificados, como pesquisas de opinião pública, censitárias, que utilizem informações de domínio público, que ver-

\section{Fluxograma de escolha dos documentos}

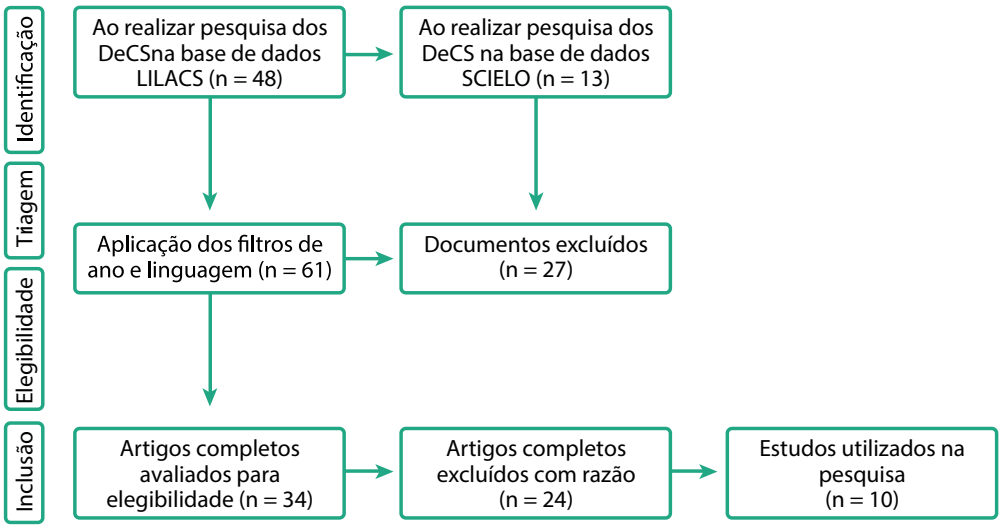

Fonte: Protocolo PRISMA sem sobre textos científicos ou que se baseiem em práticas profissionais.

\section{RESULTADOS}

Ao realizar a busca na BVS, foi filtrada apenas a base de dados LILACS, obtendo uma totalidade de 48 documentos. $\mathrm{Na}$ base de dados SciELO, 13 estudos, resultando em 61. Após a aplicação dos critérios estabelecidos, restaram 33 estudos, dos quais, apenas 10 fizeram parte da construção do estudo, dos quais, LILACS (09) e SciELO (01).

Para demonstração de todo processo de busca e escolha dos documentos utilizados, utilizou-se um fluxograma baseado no protocolo PRISMA, ilustrado abaixo.

\section{DISCUSSÃO}

A violência obstétrica é um tema que ganha cada vez mais visibilidade e gera

Tabela 1 - Artigos distribuídos por autor e ano de publicação, objetivo e principais achados

Autor e ano

Objetivo

Descrever a violência obstétrica e a assistência de enfermagem na promoção do parto seguro.

Pérez, Oliveira, Lago, 2018

Analisar as consequências da violência obstétrica na vida de mulheres que vivenciaram essa experiência.

\section{Principais achados}

Um estudo formado por gestantes e seus recém-nascidos nos distintos territórios do país, manifestar-se que, da amostra total da pesquisa de 23.940 mulheres, $56,8 \%$ foram consideradas como casos de risco obstétrico habitual.

Foi feito uma avaliação do impacto do parto no desenvolvimento de transtornos em mulheres, e destacou que entre $60 \%$ das que passaram por uma história de parto traumático 20\% apresentaram sintomas, depressivos semanas após o parto.

Analisar as condições organizacionais e OMC,2017 estruturais das instituições de saúde que podem favorecer ou dificultar a ocorrência de violência obstétrica.
Torres JA., Santos I, Vargens
Essa modalidade de violência obstétrica Também pode ser considerada institucional, uma vez que ocorre em instituições prestadoras de cuidados à saúde
Velho MB, Santos EKA, Brüggeman OM, Camargo BV, 2017

Demonstrar o papel do enfermeiro nesse processo de humanização da assistência.
Destacar a importância de minimizar até

Brasil, Ministério da Saúde, 2017 anulares definitivamente práticas abusivas $\mathrm{e}$ prejudiciais, praticadas por profissionais.
Os principais achados da humanização ao parto estão: protagonismo da mulher e suas escolhas, conscientização e capacitação com ações educativas permanentes em saúde.

0 estudo evidenciou que quanto mais a gestante dispõe de informações acerca dos procedimentos realizados, mais seus medos e ansiedades são minimizados.

No Brasil a violência obstétrica é praticada por médicos e profissionais da enfermagem, em especial, na forma de negligência, violência verbal e violência física, exceto a violência sexual. 


$\begin{array}{ccc}\text { Altaweli RF, Court C, Baron M. } 2015 & \begin{array}{c}\text { Identificar a experiência de mulheres primíparas } \\ \text { diante de possíveis casos de violência obstétrica } \\ \text { no parto normal. }\end{array} & \begin{array}{c}\text { Um estudo realizado em são Paulo demonstrou que } \\ \text { a desvalorização do parto normal é uma crescente } \\ \text { adoção de técnicas cirúrgicas e intervencionistas. }\end{array} \\ \begin{array}{c}\text { Carlos GA, Matozinhos FP, Carmo } \\ \text { JM, Manzo BF, 2015 }\end{array} & \begin{array}{c}\text { Construir o Discurso do Sujeito Coletivo de } \\ \text { Enfermeiros pós-graduandos em Enfermagem } \\ \text { Obstétrica sobre a violência obstétrica. }\end{array} & \begin{array}{c}\text { Destacam-se a existência de métodos estratégicos } \\ \text { capazes de reverter essa situação por meio de diretrizes } \\ \text { baseadas em evidências, mudanças de atitudes por } \\ \text { parte dos especialistas. }\end{array} \\ \begin{array}{c}\text { Silva MG, Marcelino MC, Rodrigues } \\ \text { LSP, TORO RC, SHIMO, A.K. 2015 }\end{array} & \begin{array}{c}\text { Analisando a percepção das parturientes acerca } \\ \text { da violência e as principais formas de violência } \\ \text { obstétrica sofrida pelas mulheres brasileiras }\end{array} & \begin{array}{c}\text { Os resultados apontaram que o desconhecimento da } \\ \text { mulher acerca de seus direitos pode contribuir para } \\ \text { a não percepção de condutas que se configuram em } \\ \text { violência }\end{array}\end{array}$

Analisar na literatura, as ações de assistência e Promoção à saúde da mulher praticada pela enPérez, BAG, Oliveira EV, lago M S, fermagem, com ênfase na fase reprodutiva, nos 2015 períodos do pré-natal, do parto e do puerpério, e considerando-se a vivência de abuso e violência obstétrica.
Cabe a equipe multidisciplinar colocar em prática os princípios da PNHPN e tratar o ser humano com toda a sua integralidade, direitos e especificidade.

Fonte: Elaboração própria.

discussões relevantes sobre os direitos das mulheres durante o processo de parto. Trata-se de uma temática abordada desde o final da década de 80 decorrentes de algumas atitudes discriminatórias e desumanas na assistência ao parto ${ }^{13}$. Aguiar enfatiza que a terminologia violência obstétrica é utilizada para descrever as diversas formas de violência ocorridas na assistência à gravidez, parto, pós-parto e puerpério ${ }^{14}$.

Dessa forma, a violência é considerada uma violação dos direitos das mulheres grávidas em processo de parturição, que inclui perda da autonomia e decisão sobre seus corpos. Neste sentido, significa apropriação dos processos reprodutivos das mulheres por alguns profissionais da saúde, através de uma atenção mecanizada, tecnicista e massificada do parto ${ }^{15-16}$.

A violência psicológica também faz parte dos fatores que ocasionam a violência obstétrica, é considerada cruel e uma das mais recorrentes no ambiente hospitalar, caracterizada por: privação de informações à parturiente acerca dos procedimentos realizados, realização de comentários ofensivos, insultuosos, discriminatórios, humilhantes ou vexatórios, tratarem a parturiente de forma grosseira, agressiva, não empática e zombeteira,

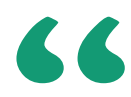

Segundo o ministério da saúde o processo de humanização do nascimento, que inclui também a possibilidade de um acompanhante à parturiente, envolve necessariamente uma mudança de atitudes humanas e nos procedimentos adotados. expor a parturiente a situações de medo e abandono.

Segundo o ministério da saúde ${ }^{17}$ o processo de humanização do nascimento, que inclui também a possibilidade de um acompanhante à parturiente, envolve necessariamente uma mudança de atitudes humanas e nos procedimentos adotados. Sendo o profissional de saúde de suma importância, por ser parte integrante da equipe que presta atenção integral a mulher, revendo conceitos, para favorecer um acolhimento completo, técnico e humano ao Cliente.

Algumas medidas durante a assistência de enfermagem são reconhecidas para a não ocorrência da violência obstétrica, por exemplo: esclarecer com uma linguagem acessível, procedimentos e ações que ajudam durante a parturição e como ela também pode colaborar para evitar a utilização de técnicas invasivas não indicadas, sempre avaliando o risco- benefício. Saber ouvir a parturiente respeitando seu momento e respeitar seu tempo para tomada de decisões, evitando constrangimentos, possibilitarem o direito de livre escolha de uma pessoa de confiança para acompanhamento durante todo pré-natal/parto, dar autonomia a mulher quanto aos seus direitos sexuais e reprodutivos, investir em aperfeiçoamen- 
to profissional e nas boas práticas baseadas em evidências ${ }^{18}$.

Neste contexto, é possível afirmar que a utilização de boas práticas de enfermagem na assistência a parturiente contribui para a humanização do parto, incentivando a mulher a retornar seu papel de protagonista, tendo a mesma o conhecimento a respeito dos seus direitos e assim reduzir as intervenções realizadas por alguns profissionais de saúde que são consideradas violência obstétrica.

\section{CONCLUSÃO}

O processo do parto é um acontecimento repleto de possíveis equívocos, condutas dolorosas e negligências, que podem gerar a violência obstétrica causando traumas físicos e psicológicos irreversíveis. Por meio desse estudo se buscou destacara importância de minimizar

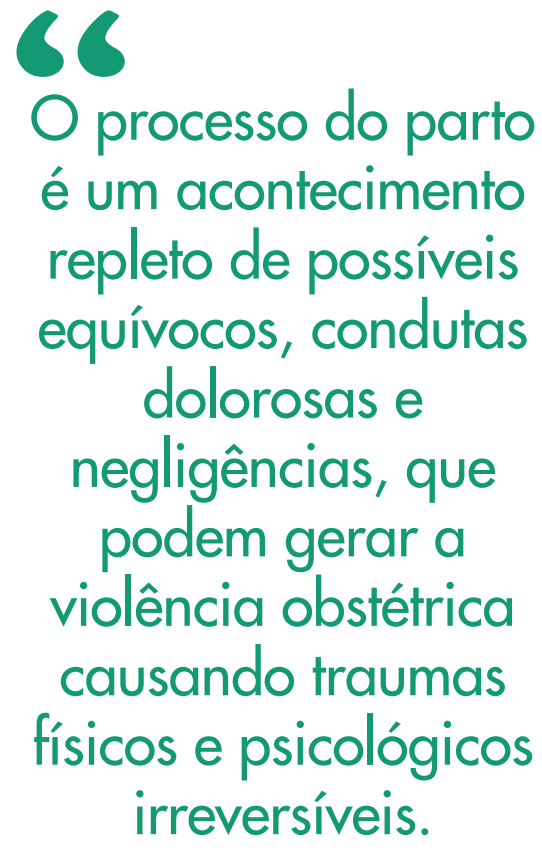

98 as práticas abusivas e prejudiciais, praticadas por alguns profissionais com o uso de estratégias que efetivem programas e políticas ao binômio mãe-filho.

É importante salientar que a capacitação dos profissionais que acompanham desde o pré-natal é primordial no acompanhamento à gestante, essas ações visam seu bem-estar físico e mental, preparando-as para a maternidade, pois uma assistência humanizada é essencial durante todo o processo de pré-parto e puerpério.

Por fim, faz-se necessário o desenvolvimento de campanhas e cursos de aprimoramento profissional, que visem o combate à violência obstétrica. É preciso que aja uma reforma na assistência prestada, baseada principalmente na humanização, respeitando a autonomia da mulher para que a mesma se sinta protagonista desse momento.

\section{Referências}

1. Velho $M B$, Santos EKA, Brüggeman $O M$, Camargo BV. Vivência do parto normal ou cesáreo: revisão integrativa sobre a percepção de mulheres. Texto Contexto Enferm. 2017. Disponivel:http://dx.doi.org/10.1590/S010407072012000200026

2. Pérez BAG, Oliveira EV, Lago MS. Percepções de Puérperas vítimas de Violência Institucional durante o Trabalho de Parto e Parto. Revista Enfermagem Contemporânea. 2015 [acesso em 18 março 2020]. Disponível: http:// dx.doi.org/10.17267/2317-3378

3. Teixeira, Neuma ZF, Pereira,Wilza R. Parto hospitalar - experiências de mulheres da periferia de Cuibá-MT. Revista Latino-Americana de Enfermagem, Brasília, v.59, n.6, nov./dec. 2015. Disponível em: http://www.scielo.br/ php.pid $=$ s00347667200600004

4. Torres JA., Santos I, Vargens OMC. Constructing a care technology conception in obstetric nursing: a sociopoetic study. Texto Contexto Enferm. 20008 DeccitedJun 5, 2017https://www.scielo.br/pdf/tce/v17n4/05.pdf

5. Andrade PON, Silva JQP, Diniz, CMM, Caminha MDE. Fatores associados à violência obstétrica na assistência ao parto vaginal em uma maternidade de alta complexidade em Recife, Pernambuco. Rev. Bras. Saúde Mater. Infant. 2016 [acesso em 16 fev 2017].https://www.scielo.br/pdf/rlae/v26/pt_01041169-rlae-26-e3069.pdf

6. Fioretti B, Paulino D. Nascer no Brasil o retrato do nascimento na voz das mulheres. Rev.Eletroncomuninfinov Saúde. 2015 [acesso em 16 Jan 2017] Disponivel: https://www.reciis.icict.fiocruz.br/index

7. Silva MG, Marcelino MC, Rodrigues LSP, TORO RC, SHIMO, A.K. Obstetricviolenceaccordingtoobstetricnurses. Northeastnetwnurs J. 2015 [acesso em 5Feb 2020]. Disponivel: http://dx.doi. org/10.15253/21756783.2014000400020

8. Cardoso FJC et al, Violência obstétrica institucional no parto: percepção de profissionais da saúde. Revista de Enfermagem, 2017. www.scielo.br/pdf/ rbfis/v11n1/12

9. Prodanov CC, Freitas EC. Metodologia do trabalho científico: Métodos e Técnicas da Pesquisa e do Trabalho Acadêmico. Universidade Free vale.
Novo Hamburgo, 2018. https://www.feevale.br/institucional/editora-feevale/metodologia-do-trabalho-cientifico

10. Galvão MCB, Ricarte ILM. Revisão sistemática da literatura: Conceituação, produção e publicação. Logeion: Filosofia da Informação, vol. 06, n. 01, pag. 57-73, 15 de Set. 2019. DOI: https://doi.org/10.21728/ logeion.2019v6n1.p57-73

11. Lakatos EM, Marconi MA. Fundamentos de metodologia científica. Atlas, 5. Ed. São Paulo, 2015. http://biblioteca.virtual.ufpb.br/files/metodologia.pdf 12. Carvalho AS et al, Violência obstétrica: a ótica sobre os princípios bioéticos e direitos das mulheres. Brazilian Journal of Surgery and Clinical Research, 2017. https://www.scielo.br/pdf/rbfis/v11n1/12.pdf

13. Pozzio MR. La gineco-obstetriciaen México: entre el "parto humanizado" y La violência obstétrica. V. estudfem. 2016 [cited 2017 Jan 28];24(1):10117. Available. https://periodicos.ufpe.br/revistas/revistaenfermagem/article/ viewFile/23523/26086

14. Aguiar JM, D'Oliveira AFL, Schraibe LB, Diniz SG. Violência institucional, autoridade médica e poder nas maternidades sob a ótica dos profissionais de saúde. Cad saúde pública.2016 .Availablefrom: http://www.scielo.br/pdf/ csp/v29n11/15.pdf

15. Brasil. Agência Nacional de Saúde Suplementar. 0 modelo de atenção obstétrica no setor de Saúde Suplementar no Brasil: cenários e perspectivas. Rio de Janeiro: ANS, 2018. https://scielosp.org/article/csp/2019.v35n3/ e0009311818

16. D'Oliveira AFPL, DINIZ CSG. Violence against women in health care institutions: an emerging problem. Lancet, 359(11), 1681-1685. 2016Doi: 10.1016/S0140-6736(02)08592

17. Ministério da Saúde. Área Técnica de Saúde da Mulher. Parto, aborto e puerpério Área Técnica da Mulher. Brasília: Ministério da Saúde. 2015. Disponível em: http://bvsms.saude.gov.br/bvs/publicacoes

18. Soares GCF,Andre TTO, Diniz CSG, Narchi NZ. Adjustment disorders in the postpartum resulting from childbirth: a descriptive and exploratory study. Online braz nurs .2012 Available from: http://www.objnursing.uff.br 